\title{
A Young Case with Multi-Infarct Dementia Associated with Lupus Anticoagulant
}

\author{
Akira Kurita, Takeo Hasunuma, Soichiro Mochio, Takao Shimada, \\ Yukihide IsOGAI and Tomoko KURAHASHI*
}

\begin{abstract}
A 39-year-old man was admitted because of an abrupt onset of right-side weakness and dysarthria. During the 2 years before admission, he had suffered from insomnia, depressed mood and progressive memory disturbance. Neurological and psychiatric examination revealed severe intellectual impairment in addition to the neurological deficits. Neuroradiological examinations revealed multiple brain infarcts. He had no risk factor for stroke except for lupus anticoagulant. He was diagnosed as having multi-infarct dementia associated with antiphospholipid antibodies. This case suggests that it is necessary to investigate antiphospholipid antibodies in addition to neuroradiological examination when relatively young patients present with unexplained cognitive or behavioral symptoms.
\end{abstract}

(Internal Medicine 33: 373-375, 1994)

Key words: antiphospholipid antibodies, brain infarction, vascular dementia

\section{Introduction}

Lupus anticoagulants (LAs) and anticardiolipin antibodies (ACLs), which are circulating antibodies directed against phospholipids (antiphospholipid antibodies: APLs), have recently been recognized as markers for an increased risk of thrombosis (1-6). Several studies have reported a high incidence of recurrent cerebrovascular ischemia in APL positive subjects $(2,3)$ including young patients $(7,8)$. APLs have also been found in elderly subjects with multi-infarct dementia (MID) (9). In one report, 2 young patients with MID were described in association with ACLs (10). However, no report to date has documented a young case with MID associated with LA. In this communication, we report a relatively young case in whom LA was the only possible factor contributing to the etiology of MID.

\section{Case Report}

A 39-year-old man was admitted to our hospital because of right-side weakness and intellectual dysfunction. Two years prior to admission, he first complained of insomnia, loss of energy and depressed mood, for which his physician prescribed antidepressants. Over the subsequent months he developed progressive memory disturbance, which finally made it impossible for him to keep his job. Two months before admission, he became disoriented, and unable to perform everyday activities. These symptoms fluctuated during the following weeks. On the first visit to our hospital, he suddenly developed right hemiparesis and dysarthria, and was therefore admitted. His medical history was unremarkable.

Physical examination on admission showed no abnormal findings. Neurological examination revealed dysarthria and right hemiparesis with bilaterally increased patellar tendon reflex. His intellectual function was markedly impaired with a Hasegawa Dementia Rating Scale score of 12 and a total IQ below 60 (verbal: 64; performance: $<60$ ) on the Wechsler Adult Intelligence Scale. The Hachinski's ischemic score (11) was 11. Routine laboratory studies including urinalysis, erythrocyte sedimentation rate, complete blood counts and blood chemistry were unremarkable. Serologic tests showed negative results for syphilis, antinuclear antibodies (ANA) and anti-DNA antibodies. Serum immunoglobulin and complement levels were normal. While ACL was not detected, LA was positive on diluted prothrombin time method in both the initial and a repeat examination with an interval of 6 weeks. Coagulation studies disclosed normal values; prothrombin time was $76 \%$ and activated partial thromboplastin time (APTT) was $35.3 \mathrm{sec}$.

From the Third Department of Internal Medicine, Jikei University School of Medicine, Tokyo, and *the Department of Internal Medicine, Jikei-Daisan Hospital, Tokyo

Received for publication November 8, 1993; Accepted for publication April 5, 1994

Reprint requests should be addressed to Dr. Akira Kurita, the Third Department of Internal Medicine, Jikei University School of Medicine, 3-25-8, NishiShinbashi, Minato-ku, Tokyo 105 
Antithrombin III and protein $\mathrm{C}$ were within normal limits. Cerebrospinal fluid (CSF) examination showed $1 \mathrm{cell} / \mathrm{mm}^{3}$, glucose $85 \mathrm{mg} / \mathrm{dl}$, protein level of $55 \mathrm{mg} / \mathrm{dl}$ and $\mathrm{IgG} 6.3 \mathrm{mg} / \mathrm{dl}$. Neither myelin basic protein nor oligoclonal band was present. Electroencephalography demonstrated occasional slow waves. Computed tomography (CT) of the brain on admission demonstrated multiple low density lesions consistent with infarcts in the basal ganglia and periventricular region with diffuse white matter lucency (Fig. 1). Magnetic resonance imaging (MRI) disclosed additional lesions including one in the left internal capsule, which was thought to be responsible for the right hemiparesis, with a high signal on $\mathrm{T}_{2}$-weighted images (Fig. 2). Cerebral angiography (4-vessel study) did not show any vascular abnormalities. These findings led to a diagnosis of MID associated with LA. After admission, he received antiplatelet therapy (sodium ozagrel; thromboxane $A_{2}$ inhibitor), which was effective for the right hemiparesis and dysarthria. Three months later, he was discharged without any paresis. However,

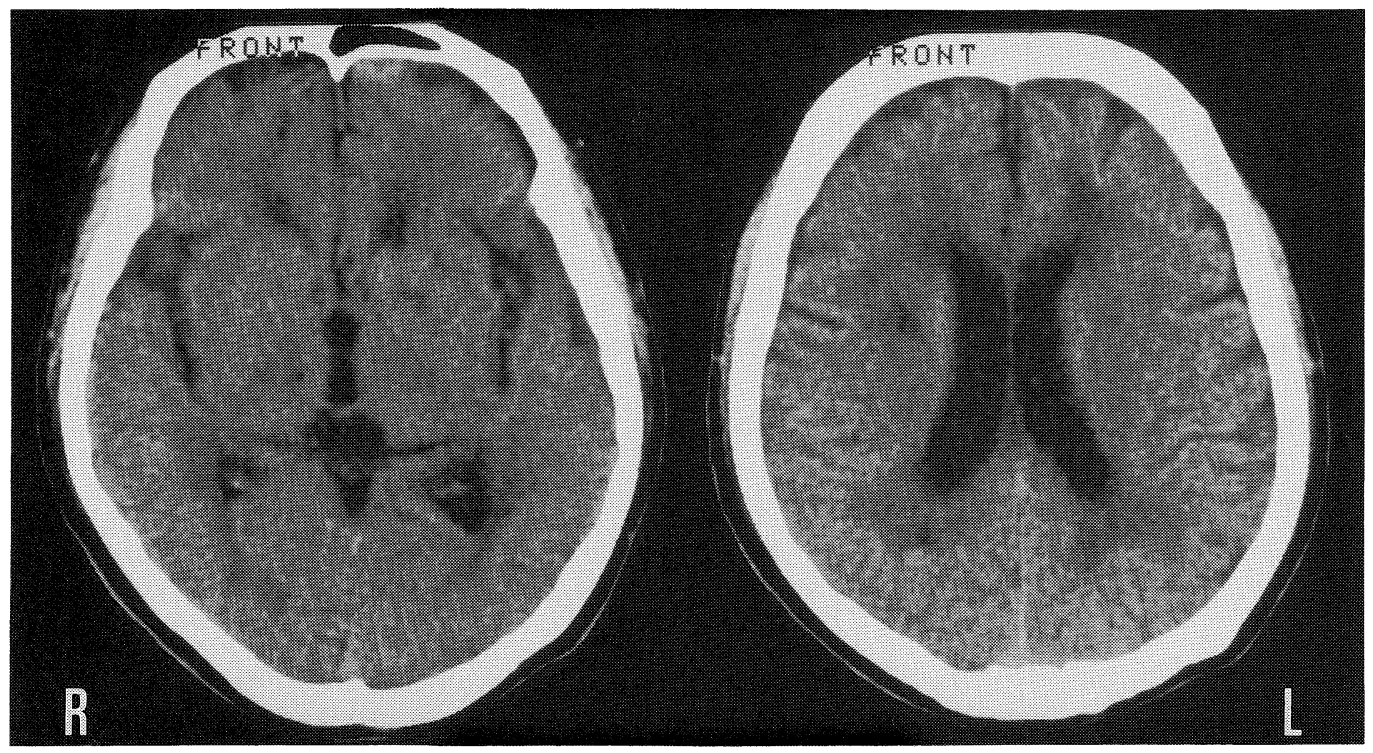

Fig. 1. CT scan of the brain on admission discloses multiple low density lesions in the basal ganglia and periventricular region with diffuse white matter lucency.

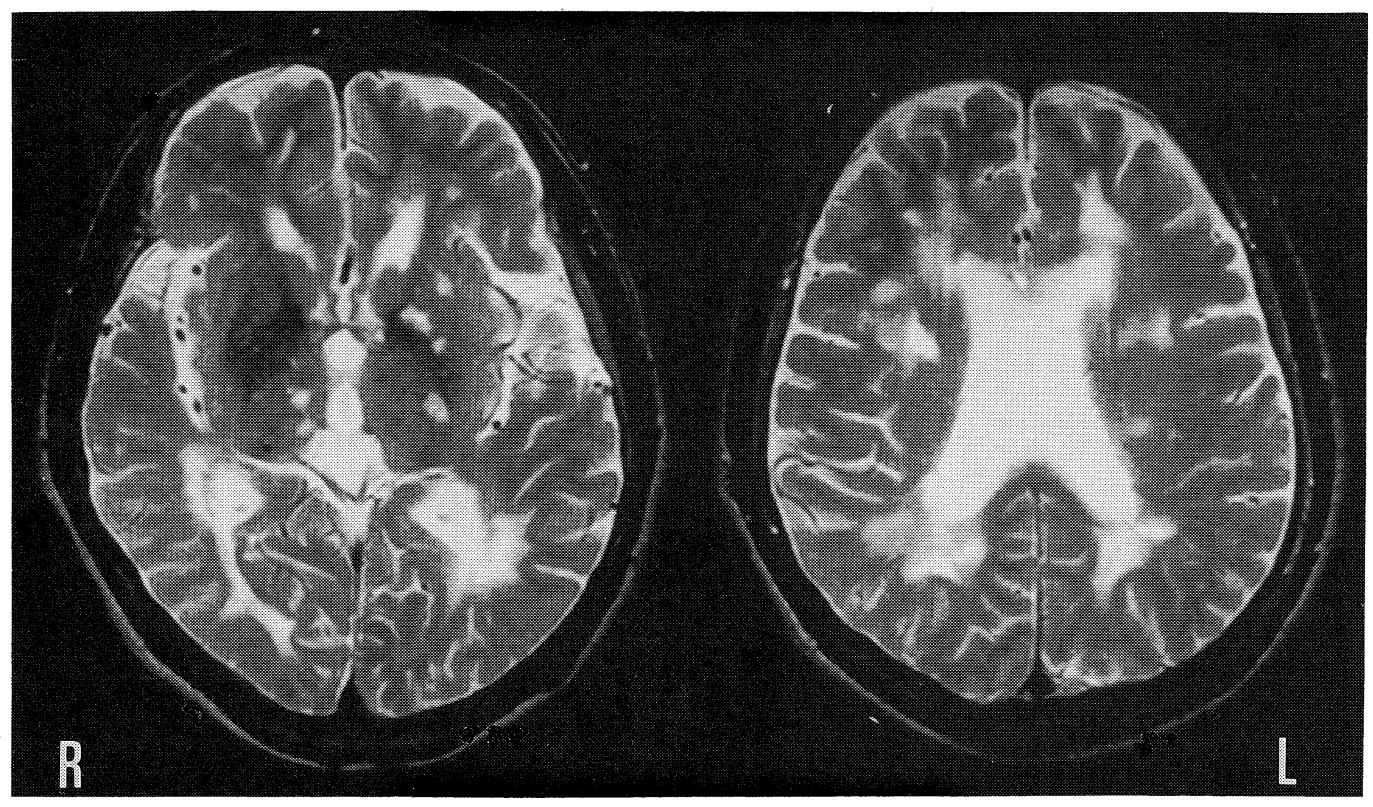

Fig. 2. Axial $\mathrm{T}_{2}$-weighted MR image (TR3000/TE80) taken 26 days after admission demonstrates multiple high signal lesions in the basal ganglia, periventricular region and left internal capsule. 


\section{MID Associated with Lupus Anticoagulant}

his intellectual impairment remained unchanged.

\section{Discussion}

For this 39-year-old man, a diagnosis of MID was made on the basis of the clinical features including neuroradiological findings (12). Since he had no stroke risk factor other than LA, it is possible to relate the infarcts to it. While mental disorders are the commonest manifestation of cerebral lupus (13), the present patient did not fulfill the 1982 American Rheumatism Association criteria for systemic lupus erythematosus (SLE) (14); other than the neurologic disorder, he had no clinical or laboratory findings characteristic of SLE. Multiple sclerosis may be another condition in the differential diagnoses of the neuroradiological abnormalities, but it is less likely. He had no optic neuritis, and CSF study did not indicate presence of demyelination. The favorable effect of the antiplatelet therapy on his focal neurological deficits may support the possibility that he had brain infarcts. Recently, several studies have found a close relation between thrombosis and APLs (1-6). While a false-positive syphilis test and a low platelet count have been understood as clues to APLs, the present patient showed neither of these signs. Levine et al suggested screening patients at any age with cerebrovascular or ocular syndromes for APLs who do not have an obvious etiology (3). Our observations are in agreement with their suggestion.

Despite the age of under 40 years, the present patient developed dementia attributable to multiple infarcts. Since he had been in good health up to the age of 37 when he first had behavioral symptoms, the onset of the illness might date back to that time. Unfortunately, an exact clinical diagnosis had not been made until he developed focal neurological deficits 2 years later. His initial symptoms, which were psychiatric rather than neurological domain, might have led to an early misdiagnosis. Recent studies have indicated that young patients with APLs are at a high risk of cerebrovascular disease $(7,8)$. Other studies have reported that most APL-positive patients have recurrent infarcts $(2,3)$. These reports together indicate that even at a young age, patients with APLs can develop MID unless they receive adequate treatment for thrombosis. In a report by Coull et al (10), 2 young patients developed MID associated with ACLs in the absence of LA. While the present patient had LA but not ACL, their report and our observations together suggest that it is imperative to investigate both LA and ACL in addition to the neuroradiological examination regardless of focal neurological deficits, in relatively young patients with unexplained cognitive or behavioral symptoms. Early detection of APLs may be the most important step to prevent the occurrence and progression of MID.

This case was presented at 420th Kanto regional meeting of Japanese Society of Internal Medicine, July 10, 1993, Tokyo.

\section{References}

1) Mueh JR, Herbst KD, Rapaport SI. Thrombosis in patients with the lupus anticoagulant. Ann Intern Med 92: 156, 1980.

2) The Antiphospholipid Antibodies in Stroke Study Group. Clinical and laboratory findings in patients with antiphospholipid antibodies and cerebral ischemia. Stroke 21: 1268, 1990.

3) Levine SR, Deegan MJ, Futrell N, Welch KMA. Cerebrovascular and neurologic disease associated with antiphospholipid antibodies: 48 cases. Neurology 40: 1181, 1990.

4) Kittner SJ, Gorelick PB. Antiphospholipid antibodies and stroke: An epidemiological perspective. Stroke 23 (suppl I): 19, 1992.

5) Hess DC. Stroke associated with antiphospholipid antibodies. Stroke $\mathbf{2 3}$ (suppl I): 23, 1992.

6) Hamada J. Antiphospholipid antibody and cerebrovascular disease. Sinkei Naika (Neurol Med) 37: 567, 1992 (in Japanese).

7) Brey RL, Hart RG, Sherman DG, Tegeler CH. Antiphospholipid antibodies and cerebral ischemia in young people. Neurology 40: 1190, 1990.

8) Nencini P, Baruffi MC, Abbate R, Massai G, Amaducci L, Inzitari D. Lupus anticoagulant and anticardiolipin antibodies in young adults with cerebral ischemia. Stroke 23: 189, 1992.

9) Kushner M, Simonian N. Lupus anticoagulants, anticardiolipin antibodies, and cerebral ischemia. Stroke 20: 225, 1989.

10) Coull BM, Bourdette DN, Goodnight SH Jr, Briley DP, Hart R. Multiple cerebral infarctions and dementia associated with anticardiolipin antibodies. Stroke 18: 1107, 1987.

11) Hachinski VC, Iliff LD, Zilhka E, et al. Cerebral blood flow in dementia. Arch Neurol 32: 632, 1975.

12) American Psychiatric Association. Diagnostic and Statistical Manual of Mental Disorders, 3rd edition-revised. American Psychiatric Association, Washington DC, 1987, p. 121.

13) Brown MM, Swash M. Systemic lupus erythematosus. in: Handbook of Clinical Neurology vol. 11 (55), Toole JF, Ed. Elsevier Science Publishers, Amsterdam, 1989, p. 369.

14) Tan EM, Cohen AS, Fries JF, et al. The 1982 revised criteria for the classification of systemic lupus erythematosus. Arthritis Rheum 25: 1271, 1982. 